\title{
ToxicDocs and the fight against biased public health science worldwide
}

\author{
Christer Hogstedt ${ }^{1} \cdot$ David H. Wegman ${ }^{2}$
}

(C) Macmillan Publishers Ltd., part of Springer Nature 2018

The existence of ToxicDocs is new to us and exciting [1]. We foresee several different areas of concern that will benefit from the availability of this resource. It seems to make possible a range of historical studies on the behavior of international industry, particularly where there are questions about the creation of questionable knowledge or the distortion and denial of scientific evidence. Such studies may shed light on the fact that it has often taken too long for public authorities to regulate chemical exposures. Delay has been blamed on 'conflicting evidence' about asbestos, benzene, phenoxy acids, and many other carcinogens, plus metals and solvents. This 'conflicting evidence' has taken the form of everything from questioning methods or the integrity of the researchers, producing the so-called negative studies by applying inadequate exposure histories or ill-defined outcomes, lobbying to influence researchers and officials in national and international research centers and in regulatory agencies [2]. We expect that many 'detective stories' will emerge from the use of this new and searchable document bank.

ToxicDocs may encourage accurate reporting of conflicts of interest. Many countries produce standardized systematic reviews of the documented scientific knowledge on toxicity of chemicals as the basis for regulations and exposure limits. One common item in the evaluation protocol for each relevant article in these reviews concerns potential risk of biased research due to industry financing or employment. In the future, when undertaking such reviews, it will be possible to check ToxicDocs to learn if there is evidence of data manipulations or other irregularities surrounding the publications included in the review.

Christer Hogstedt

christer.hogstedt@gmail.com

David H. Wegman

David_Wegman@uml.edu

1 Tullgårdsgatan 28, 11668 Stockholm, Sweden

2 University of Massachusetts at Lowell, Lowell, MA, USA 
The proliferation of open access journals, including a number that are considered predatory [3], may distort reviews. These journals, while advertised as "peerreviewed," may have questionable review processes or questionable reviewer qualifications. In the modern electronic world, the publication of research findings on risks of environmental exposures can be cited, regardless of quality. Scientists and policy makers are constantly presented with misleading 'facts.' ToxicDocs permits anyone who is concerned about research reports ready access to searchable information that can be used to challenge or substantiate questionable results.

ToxicDocs could be particularly useful in low- and middle-income countries where some industry interests likely try to introduce or prolong the use of toxic materials such as pesticides, often where the available resources for evaluating existing literature are limited. ToxicDocs could alert people in these countries to lobbying methods that have been used by industry in the US and Europe.

It is hard to imagine the full potential of ToxicDocs for studies, exposés, and stories that may result from this incredibly large collection of documents. It will obviously demand high skill in handling "Big Data" and it will be a challenge that should stimulate younger generations who are familiar with computer technology and data handling to get involved.

Specifically, we want to note how generous, honorable, and extremely important is the decision to make the use of ToxicDocs free of charge. The timing is perfect for the news announcing this remarkable data source in this era of 'fake facts.'

As with the Legacy Tobacco Documents Library (now the Truth Tobacco Industry Documents) [4], we can look forward to continuing new additions to ToxicDocs. The massive searchable data repository should continue to empower those who seek to protect our environment, including our workplaces, and to understand fully all the available information behind the science needed for action.

We would like to congratulate the creators of this extremely important data source that has been made available to researchers, mass media, public agents, and the concerned public and to express our admiration for the enormous and skillful work that has made it possible. ToxicDocs will be a strong weapon in the fight against biased public health science and corrupt lobbying in the interest of private profit.

\section{References}

1. Rosner D, Markowitz G, Chowkwanyun M. ToxicDocs (www.ToxicDocs.org): from history buried in stacks of paper to open, searchable archives online, J Public Health Pol. [special section] "ToxicDocs: Opening a new era of evidence for policies to protect public health" (Guest Eds. Rosner D, Markowitz G, Chowkwanyun M). 2018; 39(1); https://doi.org/10.1057/s41271-017-0106-8.

2. Michaels D. Doubt is their product. Oxford: Oxford University Press; 2008.

3. Sorokowski P, Kulczycki E, Sorokowska A, Pisanski K. Predatory journals recruit fake editor (Commentary). Nature. 2017;543:481-483.

4. University of California at San Francisco (UCSF). Truth Tobacco Industry Documents. https://www. industrydocumentslibrary.ucsf.edu/tobacco/. 
Christer Hogstedt, M.D., PhD is a specialist in occupational health, former Deputy Director General of the Swedish National Institute of Working Life, and senior researcher at Institute of Environmental Medicine, Karolinska Institute, Stockholm, Sweden.

David H. Wegman, M.D., M.Sc., is Professor Emeritus, University of Massachusetts Lowell, MA, USA, and Adjunct Professor, Harvard School of Public Health, Boston, MA, USA. He is a specialist in occupational epidemiology, surveillance, and policy. 